SFB 649 Discussion Paper 2008-063

\title{
Discrete-Time Stochastic Volatility Models and MCMC-Based Statistical Inference
}

\author{
Nikolaus Hautsch* \\ Yangguoyi Ou*
}

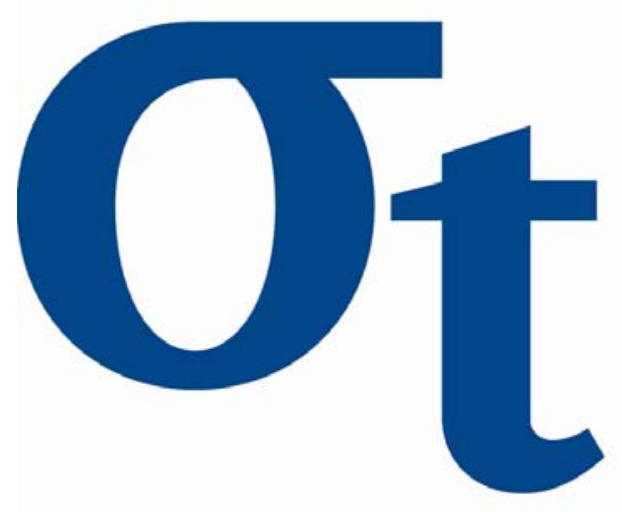

9)

$\forall$

6

* Humboldt-Universität zu Berlin, Germany

This research was supported by the Deutsche Forschungsgemeinschaft through the SFB 649 "Economic Risk". 


\title{
Discrete-Time Stochastic Volatility Models and MCMC-Based Statistical Inference *
}

\author{
Nikolaus Hautsch ${ }^{\dagger}$ \\ Humboldt-Universität zu Berlin, CASE, CFS, QPL \\ Yangguoyi $\mathrm{Ou}^{\ddagger}$ \\ Humboldt-Universität zu Berlin, CASE
}

This version: July 2008

\begin{abstract}
In this paper, we review the most common specifications of discrete-time stochastic volatility (SV) models and illustrate the major principles of corresponding Markov Chain Monte Carlo (MCMC) based statistical inference. We provide a hands-on approach which is easily implemented in empirical applications and financial practice and can be straightforwardly extended in various directions. We illustrate empirical results based on different SV specifications using returns on stock indices and foreign exchange rates.

Keywords: Stochastic Volatility, Markov Chain Monte Carlo, Metropolis-Hastings algorithm, Jump Processes
\end{abstract}

JEL Classification: C15, C22, G12

\section{Introduction}

Stochastic volatility (SV) models are workhorses for the modelling and prediction of timevarying volatility on financial markets and are essential tools in risk management, asset pricing and asset allocation. In financial mathematics and financial economics, stochastic volatility is typically modeled in a continuous-time setting which is advantageous for derivative pricing and portfolio optimization. Nevertheless, since data is typically only observable at discrete points in time, in empirical applications, discrete-time formulations of SV models are equally important.

\footnotetext{
* The authors gratefully acknowledge financial support from the EU Commission through MRTN-CT2006-034270 COMISEF as well as from the Deutsche Forschungsgemeinschaft through the SFB 649 "Economic Risk".

${ }^{\dagger}$ Institute for Statistics and Econometrics, and CASE - Center for Applied Statistics and Economics, Humboldt-Universität zu Berlin, Center for Financial Studies (CFS), Frankfurt, as well as Quantitative Products Laboratory (QPL), Berlin. Address: Spandauer Str. 1, D-10178 Berlin, Germany. Email: nikolaus.hautsch@wiwi.hu-berlin.de. Tel: +49 30 20935711, fax: +49 3020935712.

${ }^{\ddagger}$ School for Business and Economics and CASE - Center for Applied Statistics and Economics, HumboldtUniversität zu Berlin. Email: yang.ou@wiwi.hu-berlin.de. Address: Spandauer Str. 1, D-10178 Berlin, Germany.
} 
SV models can be economically motivated by the mixture-of-distribution hypothesis (MDH) postulated by Clark (1973), whereby asset returns follow a mixture of normal distributions with a mixing process depending on the (unobservable) information arrival process. If the mixing process is positively autocorrelated, the resulting return process reveals volatility clustering which is a well-known and typical feature of financial return series. The MDH gives rise to the idea that asset return volatility follows its own stochastic process which is updated by unobservable innovations. This is in contrast to an autoregressive conditional heteroscedasticity $(\mathrm{ARCH})$ model introduced by Engle (1982), where the conditional variance given the available information set is a function of past observations. Denote $h_{t}$ as the time- $t$ conditional variance of asset return $y_{t}$ with conditional mean $\mu_{t}$ and $y_{t}-\mu_{t}=h_{t}^{1 / 2} z_{t}, z_{t} \sim \operatorname{IID}(0,1)$, and let $\mathcal{F}_{t}$ denote the time- $t$ information set. Then, ARCH processes imply $\operatorname{Var}\left[h_{t} \mid \mathcal{F}_{t-1}\right]=0$, i.e., the variance is conditionally deterministic given the (observable) history of the process. Conversely, SV models can be characterized by the property $\operatorname{Var}\left[h_{t} \mid \mathcal{F}_{t-1}\right] \neq 0$, i.e., there is an unpredictable component in $h_{t}$.

A main difficulty of the SV framework compared to the widely used (Generalized) ARCH model is that the likelihood of SV models is not directly available. This requires the use of simulation techniques, like simulated maximum likelihood, method of simulated moments or Markov chain Monte Carlo (MCMC) techniques. Because of the computational costs, SV models are still less popular in financial practice. Nevertheless, increasing computer power and the further development of efficient sampling techniques weaken this drawback noticeably. Furthermore, recent literature on the estimation of realized volatility confirms the idea of the MDH that log returns follow a normal - log normal mixture (see, e.g., Andersen, Bollerslev, Diebold \& Labys (2003)) and thus strengthens the economic foundation of the SV model. Finally, SV models provide a natural framework to accommodate specific properties of financial return processes such as fat-tailedness, leverage effects and the occurrence of jumps.

The main objective of this study is to present the most important specifications of discrete-time SV models, to illustrate the major principles of Markov Chain Monte Carlo (MCMC) based statistical inference, and to show how to implement these techniques to estimate SV models. In this context, we provide a hands-on approach which is easily extended in various directions. Moreover, we will illustrate empirical results based on different SV specifications using returns on stock indices and foreign exchange rates.

In Section 2, we will introduce the standard SV model. Section 3 presents several extended SV models. MCMC based Bayesian inference is discussed in Section 4, whereas empirical illustrations are given in Section 5 . 


\section{The Standard Stochastic Volatility Model}

The standard stochastic volatility model as introduced by Taylor (1982) is given by

$$
\begin{array}{ll}
y_{t}=\exp \left(h_{t} / 2\right) u_{t}, & u_{t} \sim \mathrm{N}(0,1), \\
h_{t}=\mu+\phi\left(h_{t-1}-\mu\right)+\eta_{t}, & \eta_{t} \sim \mathrm{N}\left(0, \sigma_{\eta}^{2}\right),
\end{array}
$$

where $y_{t}$ denotes the $\log$ return at time $t, t=1, \ldots, T$, and $h_{t}$ is the log volatility which is assumed to follow a stationary $\operatorname{AR}(1)$ process with persistence parameter $|\phi|<1$. The error terms $u_{t}$ and $\eta_{t}$ are Gaussian white noise sequences. The unconditional distribution of $h_{t}$ is given by

$$
h_{t} \sim \mathrm{N}\left(\mu_{h}, \sigma_{h}^{2}\right), \quad \mu_{h}=\mu, \quad \sigma_{h}^{2}=\frac{\sigma_{\eta}^{2}}{1-\phi^{2}}
$$

where $\mu_{h}$ and $\sigma_{h}^{2}$ denote the unconditional mean and variance of returns, respectively.

Under the assumption that $\mathrm{E}\left[y_{t}^{4}\right]<\infty$, the first two even moments of $y_{t}$ are given by

$$
\begin{aligned}
& \mathrm{E}\left[y_{t}^{2}\right]=\mathrm{E}\left[\exp \left(h_{t}\right)\right] \mathrm{E}\left[u_{t}^{2}\right]=\exp \left(\mu_{h}+\sigma_{h}^{2} / 2\right), \\
& \mathrm{E}\left[y_{t}^{4}\right]=\mathrm{E}\left[\exp \left(2 h_{t}\right)\right] \mathrm{E}\left[u_{t}^{4}\right]=3 \exp \left(2 \mu_{h}+2 \sigma_{h}^{2}\right) .
\end{aligned}
$$

Consequently, the kurtosis is

$$
\mathrm{K}\left(y_{t}\right) \stackrel{\text { def }}{=} \frac{\mathrm{E}\left[y_{t}^{4}\right]}{\mathrm{E}\left[y_{t}^{2}\right]^{2}}=3 \exp \left(\sigma_{h}^{2}\right)=3 \exp \left(\frac{\sigma_{\eta}^{2}}{1-\phi^{2}}\right)
$$

with $\mathrm{K}\left(y_{t}\right)>3$ as long as $\sigma_{\eta}^{2}>0$. Hence, the kurtosis generated by SV processes increases with $\sigma_{\eta}^{2}$ and $|\phi|$ (given $|\phi|<1$ ).

The autocorrelation function $(\mathrm{ACF})$ of $y_{t}^{2}$ is computed as

$$
\operatorname{Corr}\left(y_{t}^{2}, y_{t-\tau}^{2}\right)=\frac{\exp \left(\sigma_{h}^{2} \phi^{\tau}\right)-1}{3 \exp \left(\sigma_{h}^{2}\right)-1}, \quad \tau=1,2, \ldots,
$$

and thus decays exponentially in $\tau$. Consequently, for $\phi \in(0,1)$, squared returns are positively autocorrelated.

The estimation of SV models is not straightforward since the likelihood cannot be computed in closed form. Let $\theta$ denote the collection of all model parameters, e.g., $\theta=\left(\mu, \phi, \sigma_{\eta}^{2}\right)$ for the standard SV model. Then, the likelihood function is defined by

$$
p(y \mid \theta) \stackrel{\text { def }}{=} \int_{h} p(y \mid h, \theta) p(h \mid \theta) d h,
$$

where $y=\left(y_{1}, \ldots, y_{T}\right)$ and $h=\left(h_{1}, \ldots, h_{T}\right)$ are the vectors of returns and latent volatility states, respectively. The so-called full-information likelihood, corresponding to the conditional probability density function (p.d.f.), $p(y \mid h, \theta)$, is specified by $1 \mathrm{a}$, whereas the 
conditional p.d.f. of the volatility states, $p(h \mid \theta)$, is given by $1 \mathrm{~b}$. The likelihood function (7) is an analytically intractable $T$-dimensional integral with respect to the unknown latent volatilities. In the econometric literature, several estimation methods have been proposed, including generalized method of moments (Melino \& Turnbull 1990), quasi-maximum likelihood estimation (Harvey, Ruiz, \& Shephard 1994), efficient method of moments (Gallant, Hsie, \& Tauchen 1997), simulated maximum likelihood (Danielsson 1994) and efficient importance sampling (Liesenfeld \& Richard 2003). Markov Chain Monte Carlo (MCMC) techniques have been introduced by Jacquier, Polson, \& Rossi (1994) and Kim, Shephard, \& Chib (1998). More details on MCMC-based inference will be given in Section 4.

\section{Extended SV Models}

\subsection{Fat Tails and Jumps}

Though the standard SV model is able to capture volatility clustering typically exhibited by financial and economic time series, the model implied kurtosis is often far too small to match the sample kurtosis observed in most financial return series. See, for example, Liesenfeld \& Jung (2000) and Chib, Nardari, \& Shephard (2002). An obvious reason is that a normal - log normal mixture as implied by the standard SV model is not flexible enough to capture the fat-tailedness commonly observed in financial return distributions. A further reason is that the basic SV model cannot account for potential jumps in the return process.

In this section, we discuss two SV specifications taking into account both pitfalls. The first one is an extension of the standard SV model allowing the error term $u_{t}$ to be Student- $t$ distributed resulting in the so-called SVt model. In the second approach, a jump component is introduced in the measurement equation in (1). This will lead to the so-called SVJ model.

\subsubsection{The SVt Model}

The SVt model is specified by

$$
\begin{array}{ll}
y_{t}=\exp \left(h_{t} / 2\right) u_{t}, & u_{t} \sim t_{v}, \\
h_{t}=\mu+\phi\left(h_{t-1}-\mu\right)+\eta_{t}, & \eta_{t} \sim \mathrm{N}\left(0, \sigma_{\eta}^{2}\right),
\end{array}
$$

where $u_{t}$ follows a standardized $t$-distribution with $v>2$ degrees of freedom. The model can be alternatively represented by a scale mixture of normal distributions. Let $\lambda_{t}$ denote an i.i.d. random variable following an inverse-gamma distribution. Then, the SVt model can be rewritten as

$$
\begin{array}{rlrl}
y_{t} & =\exp \left(h_{t} / 2\right) \sqrt{\lambda_{t}} u_{t}, & u_{t} & \sim \mathrm{N}(0,1), \\
h_{t} & =\mu+\phi\left(h_{t-1}-\mu\right)+\eta_{t}, & & \eta_{t} \sim \mathrm{N}\left(0, \sigma_{\eta}^{2}\right), \\
\lambda_{t} & \sim \operatorname{Inv}-\operatorname{Gamma}(v / 2, v / 2), & v>2,
\end{array}
$$


where $\lambda_{t}$ itself is a latent variable. The representation of the SVt model in terms of a scale mixture is particularly useful in an MCMC context since it converts a non-log-concave sampling problem into a log-concave one. This allows for sampling algorithms which guarantee convergence in finite time, see ,e.g., Frieze, Kannan \& Polson (1994).

Allowing log returns to be Student-t distributed naturally changes the behavior of the stochastic volatility process. In the standard SV model, large values of $\left|y_{t}\right|$ induce large values of $h_{t}$. In contrast, with an additional source of flexibility, $\lambda_{t}$, the SVt model can caputure large values of $\left|y_{t}\right|$ without necessarily increasing $h_{t}$. A tpyical consequence is that SVt models imply a higher persistence in volatility dynamics than the standard SV model.

Employing simulated maximum likelihood methods Liesenfeld \& Jung (2000) provide an estimate $\hat{\nu}=6.31$ for the USD/DM foreign exchange (FX) rate from 1980 to 1990, and a value of 6.30 for the USD/JPY FX rate over 5 years from 1981 to 1985 . Chib et al. (2002) estimate the SVt model based on MCMC techniques and report an estimate $\hat{\nu}=12.53$ for daily S\&P 500 returns between July 1962 and August 1997.

\subsubsection{The SV Model with Jump Components}

The question of to which extent asset return processes are driven by continuous and/or jump components is an ongoing topic in the current literature. Both $(\mathrm{G}) \mathrm{ARCH}$ and standard SV models rest on the assumption of a continuous price process and thus are not able to accommodate jumps in returns. The latter is particularly important during periods of news arrivals when the market gets under stress and becomes less liquid. However, the SV framework allows for a natural inclusion of a jump component in the return process. This yields the SVJ model given by

$$
\begin{array}{rlrl}
y_{t} & =k_{t} q_{t}+\exp \left(h_{t} / 2\right) u_{t}, & & u_{t} \sim \mathrm{N}(0,1), \\
h_{t} & =\mu+\phi\left(h_{t-1}-\mu\right)+\eta_{t}, & & \eta_{t} \sim \mathrm{N}\left(0, \sigma_{\eta}^{2}\right), \\
k_{t} & \sim \mathrm{N}\left(\alpha_{k}, \beta_{k}\right), & \\
q_{t} & \sim \mathbb{B}(\kappa), &
\end{array}
$$

where $q_{t}$ is a Bernoulli random variable taking on the value one whenever a jump occurs with probability $\kappa$, and is zero otherwise. The jump size is represented by the time-varying random variable $k_{t}$ which is assumed to follow a normal distribution with mean $\alpha_{k}$ and variance $\beta_{k}$. Both $q_{t}$ and $k_{t}$ are latent variables. Then, the model is based on three latent components, $h_{t}, q_{t}$, and $k_{t}$.

As in the SVt model, the inclusion of a jump component influences the properties of the stochastic volatility process. Large values of $\left|y_{t}\right|$ are now attributed rather to the the 
jump component than to the volatility process. As in the SVt model this typically induces a higher persistence in the volatility process.

Eraker, Johannes, \& Polson (2003) estimate the number of jumps in returns to be approximately 1.5 per year for daily S\&P 500 returns from 1980 to 1999, and 4.4 per year for NASDAQ 100 index returns from 1985 to 1999. Chib et al. (2002) estimate 0.92 jumps per year for daily S\&P 500 returns covering a period from 1962 to 1997.

Similarly, jump components can be also included in the volatility process in order to capture instantaneous movements in volatility. Bates (2000) and Duffie, Pan, \& Singleton (2000) provide evidence that both jumps in returns and volatilities are important to appropriately capture the dynamics in financial return processes. For S\&P 500 returns from 1980 to 1999, Eraker et al. (2003) estimate 1.4 volatility jumps per year.

\subsection{The Relationship Between Volatility and Returns}

Studying the relation between expected stock returns and expected variance is a fundamental topic in financial economics. Though a positive relationship between expected returns and expected variances is consistent with the notion of rational risk-averse investors requiring higher expected returns as a risk premium during volatile market periods, it is not consistently supported by empirical research. Whereas French, Schwert, \& Stambaugh (1987) and Campbell \& Hentschel (1992) find positive relationships between expected risk premia and conditional volatility, several other studies find converse dependencies. In fact, there is evidence that unexpected returns and innovations to the volatility process are negatively correlated. This can be explained either by the volatility feedback theory by French et al. (1987), or by the well-known leverage effect discussed by Black (1976).

In this section, we will discuss two types of SV models allowing the return and volatility process to be correlated, namely the SV-in-Mean (SVM) model and the Asymmetric SV (ASV) model. While the SVM model includes the volatility component directly in the mean equation, the ASV model allows for mutual correlations between return and volatility innovations.

\subsubsection{The SV-in-Mean Model}

The SV-in-Mean (SVM) model is given by

$$
\begin{array}{ll}
y_{t}=d \cdot h_{t}+\exp \left(h_{t} / 2\right) u_{t}, & u_{t} \sim \mathrm{N}(0,1), \\
h_{t}=\mu+\phi\left(h_{t-1}-\mu\right)+\eta_{t}, & \eta_{t} \sim \mathrm{N}\left(0, \sigma_{\eta}^{2}\right),
\end{array}
$$


where the parameter $d$ captures the relationship between returns and both expected as well as unexpected volatility components. This can be seen by rewriting (14a) as

$$
y_{t}=d \cdot h_{t \mid t-1}+d\left(h_{t}-h_{t \mid t-1}\right)+\exp \left(h_{t} / 2\right) u_{t}
$$

where $h_{t \mid t-1}$ denotes the expected volatility defined by the conditional variance at time $t$ given the information available at time $t-1$. Accordingly, the term $\left(h_{t}-h_{t \mid t-1}\right)$ gives the innovation to the volatility process.

French et al. (1987) regress monthly excess returns of U.S. stock portfolios on both expected and unexpected volatility components stemming from ARMA models based on daily data. Excluding the unexpected volatility component results in a weakly positive relationship between excess returns and volatility. In contrast, including both volatility components does not only result in a significantly negative impact of the volatility innovation but also reverses the sign of the ex ante relationship. Hence, the negative relationship between unexpected returns and innovations to the volatility process seems to dominate the weaker, presumably positive, relation between the expected components.

\subsubsection{The Asymmetric SV Model}

Empirical evidence for 'good' and 'bad' news having different effects on the future volatility is typically referred to as the leverage or asymmetric effect. According to the leverage effect, an unexpected drop in prices ('bad' news) increases the expected volatility more than an unexpected increase ('good' news) of similar magnitude. According to Black (1976) this is due to asymmetric effects of changes of the firm's financial leverage ratio. In SV models, leverage effects are captured by allowing the observation error $u_{t}$ and the future process error $\eta_{t+1}$ to be correlated. Then, the ASV model is specified by

$$
\begin{aligned}
y_{t} & =\exp \left(h_{t} / 2\right) u_{t}, \\
h_{t} & =\mu+\phi\left(h_{t-1}-\mu\right)+\eta_{t}, \\
\left(\begin{array}{c}
u_{t} \\
\eta_{t+1}
\end{array}\right) & \sim \mathrm{N}\left\{\left(\begin{array}{l}
0 \\
0
\end{array}\right),\left(\begin{array}{cc}
1 & \rho \sigma_{\eta} \\
\rho \sigma_{\eta} & \sigma_{\eta}
\end{array}\right)\right\},
\end{aligned}
$$

where $\rho$ denotes the correlation between $u_{t}$ and $\eta_{t+1}$.

The ASV model has been extensively studied in the literature. Harvey \& Shephard (1996) estimate the model using quasi-maximum likelihood providing $\hat{\rho}=-0.66$ for daily U.S. stock returns ranging from 1962 to 1987. Based on the same data, Sandmann \& Koopman (1998) and Jacquier, Polson, \& Rossi (2004) estimate an ASV specification, where the contemporaneous return and volatility are correlated. Using simulated MLE methods and MCMC based Bayesian inference, the two studies provide estimates of $\hat{\rho}=-0.38$ and $\hat{\rho}=-0.48$, respectively. 


\subsection{The Long Memory SV Model}

In the previous sections, we have considered a first order autoregressive process for the log volatility $h_{t}$. This induces that the autocorrelations of $h_{t}$ decay geometrically and volatility is said to exhibit short memory. However, empirical autocorrelations for absolute and squared returns typically decay more slowly and thus are not geometrically bounded. This implies so-called long range dependence or long memory effects. See, for example, Bollerslev \& Mikkelsen (1996). One possibility to capture such effects is to allow for fractionally integrated processes, which have been developed and extensively studied over the last 25 years, see, e.g., Granger \& Joyeux (1980), and Beran (1994), among others. Long memory SV models have been introduced by Breidt, Carto, \& de Lima (1998), Harvey (1998), and Arteche (2004). Then, the log volatility process follows an $\operatorname{ARFIMA}(p, d, q)$ process given by

$$
\begin{array}{cr}
y_{t}=\exp \left(h_{t} / 2\right) u_{t}, & u_{t} \sim \mathrm{N}(0,1), \\
\phi(L)(1-L)^{d}\left(h_{t}-\mu\right)=\theta(L) \eta_{t}, & \eta_{t} \sim \mathrm{N}\left(0, \sigma_{\eta}^{2}\right),
\end{array}
$$

where $d$ denotes the fractional differencing parameter and $L$ denotes the lag operator with

$$
\phi(L)=1-\sum_{i=1}^{p} \phi_{i} L^{i}, \quad \theta(L)=1+\sum_{i=1}^{q} \theta_{i} L^{i},
$$

and the roots of the polynomials $\phi(\cdot)$ and $\theta(\cdot)$ lying strictly outside the unit circle. If $d \in(-0.5,0.5)$, the volatility process reveals long memory and is weakly stationary. The fractional differencing operator $(1-L)^{d}$ can be expressed in terms of the series expansion

$$
(1-L)^{d}=\sum_{k=0}^{\infty} \frac{\Gamma(d+1)}{\Gamma(k+1) \Gamma(d-k+1)}(-1)^{k} L^{k},
$$

with $\Gamma(\cdot)$ denoting the gamma function (see, e.g., Beran (1994)).

The autocorrelation of $\log h_{t}^{2}$ is derived, e.g., by Baillie (1996), Breidt et al. (1998), or Harvey (1998). It is asymptotically proportional to $\pi^{2 d-1}$, as long as $d \in(-0.5,0.5)$. Similar asymptotic results are applicable to $\left|y_{t}\right|$ and $y_{t}^{2}$.

Breidt et al. (1998) estimate the Fractionally Integrated SV (FISV) model by maximizing the spectral quasi-likelihood and obtain estimates of $d=0.44$ and $\phi=0.93$ for daily returns of a value-weighted market portfolio of U.S. stocks between 1962 and 1989. Gallant et al. (1997) use efficient method of moments techniques to provide estimates of $d$ ranging between 0.48 and 0.55 for a series of daily returns from the $\mathrm{S} \& \mathrm{P}$ composite price index ranging from 1928 to 1987. Brockwell (2005) develops an MCMC sampling algorithm for the estimation of the FISV model and provides $d=0.42$ for daily ASD-USD FX rates between 1999 and 2004. 


\section{MCMC-Based Bayesian Inference}

In this section, we will give a brief review of MCMC-based Bayesian inference and will illustrate its application to estimate the standard SV model. For an introduction to Bayesian econometrics, see, for example, Koop (2006) and Greenberg (2008).

\subsection{Bayes' Theorem and the MCMC Algorithm}

Let $\theta$ denote a vector of model parameters including all latent variables, and let $y$ collect the observed data. By considering $\theta$ to be a random vector, its inference is based on the posterior distribution, $p(\theta \mid y)$, which can be represented by Bayes' theorem

$$
p(\theta \mid y) \propto p(y \mid \theta) p(\theta)
$$

where $p(y \mid \theta)$ denotes the likelihood function depending on the model parameters and the data $y$. Correspondingly, $p(\theta)$ defines the prior distribution reflecting subjective prior beliefs on the distribution of $\theta$. Consequently, the posterior distribution $p(\theta \mid y)$ can be viewed as a combination of objective and subjective information. If the prior is noninformative, Bayesian inference for the parameter vector $\theta$ is equivalent to likelihood-based inference.

The principle of MCMC-based Bayesian inference is to simulate $p(\theta \mid y)$ based on a Markov chain of random draws stemming from a family of candidate-generating densities from which it is easy to sample. Let $x \in \mathbb{R}^{d}$ denote a random variable (in the given context it corresponds to $\theta$ ) following a Markov chain with transition kernel $p(x, y)$ corresponding to the conditional density of $y$ given $x$. The invariant distribution is given by $\pi^{*}(y)=\int_{\mathbb{R}^{d}} p(x, y) \pi^{*}(x) d x$. An important result in Markov chain theory is that if $p(x, y)$ satisfies the reversibility condition

$$
f(x) p(x, y)=f(y) p(y, x)
$$

then, $f(\cdot)$ is the invariant density for the kernel $p(\cdot)$, i.e., $f(\cdot)=\pi^{*}(\cdot)$.

An important MCMC technique is the Metropolis-Hastings (M-H) algorithm as developed by Metropolis, Rosenbluth, Rosenbluth, Teller, \& Teller (1953) and generalized by Hastings (1970). The major idea is to build on (22) and finding a reversible kernel whose invariant distribution equals the target distribution $f(\cdot)$. This is performed by starting with an irreversible kernel (proposal density) $q(y, x)$ for which $f(x) q(x, y)>f(y) q(y, x)$, i.e., loosely speaking, the process moves from $x$ to $y$ too often and from $y$ to $x$ too rarely. This can be corrected by introducing a probability $\alpha(x, y)<1$ that the move is made. I.e., we choose $\alpha(x, y)$ such that

$$
f(x) \alpha(x, y) q(x, y)=f(y) \alpha(y, x) q(y, x) .
$$


- For $g=1, \ldots, G$ :

1. Generate $Y$ from $q\left(x^{(j)}, y\right)$ and $U$ from $\mathbb{U}[0,1]$.

2. If $U \leq \alpha\left(x^{(j)}, Y\right)=\min \left\{\frac{f(Y) q\left(Y, x^{(j)}\right)}{f\left(x^{(j)}\right) q\left(x^{(j)}, Y\right)}, 1\right\}$

Set $x^{(j+1)}=Y$.

Else

Set $x^{(j+1)}=x^{(j)}$.

3. Return $\left\{x^{(1)}, x^{(2)}, \ldots, x^{(G)}\right\}$.

Figure 1: The Metropolis-Hasings Sampling Algorithm

It is easily shown that this relationship is fulfilled for

$$
\alpha(x, y)= \begin{cases}\min \left\{\frac{f(y) q(y, x)}{f(x) q(x, y)}, 1\right\}, & \text { if } f(x) q(x, y) \neq 0 \\ 0, & \text { otherwise. }\end{cases}
$$

This yields a transition kernel $q_{M H}(x, y)$ satisfying the reversibility condition and is defined by

$$
q_{M H}(x, y) \stackrel{\text { def }}{=} q(x, y) \alpha(x, y), \quad x \neq y
$$

The resulting $\mathrm{M}-\mathrm{H}$ sampling algorithm is summarized by figure 1 .

A crucial issue is an appropriate choice of the family of candidate-generating densities. Depending on the form and the complexity of the sampling problem, various techniques have been proposed in the literature. The probably most straightforward technique is proposed by Metropolis, Rosenbluth, Rosenbluth, Teller, \& Teller (1953) suggesting a random walk chain, where $q(x, y)=q_{0}(y-x)$, and $q_{0}(\cdot)$ is a multivariate density. Then, $y$ is drawn from $y=x+z$ with $z$ following $q_{0}$. If $q_{0}$ is symmetric around zero, we have $q(x, y)=q(y, x)$ and thus $\alpha(x, y)=f(y) / f /(x)$. A further simple choice of candidate-generating densities is proposed by Hastings (1970) and is given by $q(x, y)=q_{0}(y)$, i.e., $y$ is sampled independently from $x$ resulting in an independence chain. Then, $\alpha(x, y)=f(y) / f(x) \cdot q(x) / q(y)$. A popular and more efficient method is the acceptance-rejection (A-R) M-H sampling method which is available whenever the target density is bounded by a density from which it is easy to sample. If the target density is fully bounded, the $\mathrm{M}-\mathrm{H}$ algorithm is straightforwardly combined with an acceptance-rejection step. This principle will be illustrated in more detail in the next section in order to sample the latent volatility states $h_{t}$. A more sophisticated M$\mathrm{H}$ A-R algorithm which does not need a blanketing function but only a pseudo-dominating density is proposed by Tierney (1994).

If the dimension of $x$ is high, the M-H algorithm is facilitated by applying it to blocks of parameters. For instance, if the target density can be expressed in terms of two blocks of variables, i.e., $f\left(x_{1}, x_{2}\right)$, the M-H algorithm allows to sample from each block $x_{i}$ given the other block $x_{j}, j \neq i$. Then, the probability for moving from $x_{1}$ to the candidate value 
$Y_{1}$ given $x_{2}$ is

$$
\alpha\left(x_{1}, Y_{1} \mid x_{2}\right)=\frac{f\left(Y_{1}, x_{2}\right) q_{1}\left(Y_{1}, x_{1} \mid x_{2}\right)}{f\left(x_{1}, x_{2}\right) q_{1}\left(x_{1}, Y_{1} \mid x_{2}\right)} .
$$

If the kernel $q_{1}\left(x_{1}, Y_{1} \mid x_{2}\right)$ is the conditional distribution $f\left(x_{1} \mid x_{2}\right)$, then

$$
\alpha\left(x_{1}, Y_{1} \mid x_{2}\right)=\frac{f\left(Y_{1}, x_{2}\right) f\left(x_{1} \mid x_{2}\right)}{f\left(x_{1}, x_{2}\right) f\left(Y_{1} \mid x_{2}\right)}=1
$$

since $f\left(Y_{1} \mid x_{2}\right)=f\left(Y_{1}, x_{2}\right) / f\left(x_{2}\right)$ and $f\left(x_{1} \mid x_{2}\right)=f\left(x_{1}, x_{2}\right) / f\left(x_{2}\right)$. If $f\left(x_{1} \mid x_{2}\right)$ is available for direct sampling, the resulting algorithm is referred to as the Gibbs sampler, see (Geman \& Geman 1984).

Applying the M-H (or Gibbs) algorithm to sub-blocks of the vector $x$ is a common proceeding in Bayesian statistics if the posterior distribution is of high dimension. This is particularly true for SV models where $\theta$ also includes the unobservable volatility states. In this context, the posterior distribution $p(\theta \mid y)$ is broken up into its complete conditional distributions $p\left(\theta_{i} \mid \theta_{-i}, y\right), i=1, \ldots, N$, where $N$ is the number of conditional distributions, $\theta_{i}$ denotes the $i$-th block of parameters and $\theta_{-i}$ denotes all elements of $\theta$ excluding $\theta_{i}$. The theoretical justification for this proceeding is given by the theorem by Hammersley \& Clifford (71) which is proven by Besag (1974). The intuition behind this theorem is that the knowledge of the complete set of conditional posterior distributions,

$$
\begin{aligned}
& p\left(\theta_{1} \mid \theta_{2}, \theta_{3}, \ldots, \theta_{k}, y\right), \\
& p\left(\theta_{2} \mid \theta_{1}, \theta_{3}, \ldots, \theta_{k}, y\right), \\
& \quad \vdots \\
& p\left(\theta_{k} \mid \theta_{1}, \theta_{2}, \ldots, \theta_{k-1}, y\right),
\end{aligned}
$$

up to a constant of proportionality, is equivalent to the knowledge of the posterior distribution $p\left(\theta_{1}, \ldots, \theta_{k} \mid y\right)$. This allows applying the $\mathrm{M}-\mathrm{H}$ algorithm to sub-blocks of $\theta$ leading to the Gibbs sampler if the individual conditional posterior distributions $p\left(\theta_{i} \mid \theta_{-i}, y\right)$ are directly available for sampling. In practice, Gibbs and M-H algorithms are often combined resulting in "hybrid" MCMC procedures as also illustrated in the next section.

The implementation of MCMC algorithms involves two steps. In the first step, M-H algorithms generate a sequence of random variables, $\left\{\theta^{(i)}\right\}_{i=1}^{G}$, converging to the posterior distribution $p(\theta \mid y)$. The algorithm is applied until convergence is achieved. In practice, the convergence of the Markov chain can be checked based on trace plots, autocorrelation plots or convergence tests, such as Geweke's Z-score test, Heidelberg-Welch's stationarity test and the half-width test, see, e.g., Cowles \& Carlin (1996). In the second step, Monte Carlo methods are employed to compute the posterior mean of the parameters. In particular, given 
the generated Markov chain, $\left\{\theta^{(g)}\right\}_{g=1}^{G}$, the population mean $\mathrm{E}[f(\theta) \mid y]=\int f(\theta) p(\theta \mid y) d \theta$ can be consistently estimated by the sample mean

$$
\frac{1}{G-g_{1}} \sum_{g=g_{1}+1}^{G} f\left(\theta^{(g)}\right)
$$

where $g_{1}$ is the number of burn-in periods which are discarded to reduce the influence of initial values $\left(\theta^{(0)}\right)$. The length of the burn-in period typically consists of $10 \%-15 \%$ of all MCMC iterations.

Consequently, the implementation of MCMC techniques requires both the convergence of the Markov chain and the convergence of the sample average. If the Markov chain is irreducible, aperiodic and positive recurrent, the Markov chain $\left\{\Theta^{(g)}\right\}_{g=1}^{G}$ generated from the MCMC algorithm converges to its invariant distribution, i.e.

$$
\theta^{(g)} \stackrel{\mathcal{L}}{\rightarrow} \theta \quad \text { for } g \rightarrow \infty
$$

where $\theta \sim p(\theta \mid y)$. For more details, see, e.g., Tierney (1994) or Greenberg (2008).

The convergence of the sample average of a function $m(\cdot)$ of $\left\{\Theta^{(g)}\right\}_{g=1}^{G}$ to its population counterpart,

$$
\frac{1}{G} \sum_{g=1}^{G} m\left(\theta^{(g)}\right) \stackrel{\text { a.s. }}{\rightarrow} E[m(\theta) \mid y] \quad \text { for } G \rightarrow \infty
$$

is ensured by the ergodicity of the Markov chain. As shown by Tierney (1994), the latter property is sufficient to ensure also the convergence of the Markov chain to its invariant distribution.

\subsection{MCMC-Based Estimation of the Standard SV Model}

In this section, we will illustrate the estimation of the standard SV model using the M-H algorithm. For convenience, we restate model (1) as given by

$$
\begin{array}{ll}
y_{t}=\exp \left(h_{t} / 2\right) u_{t}, & u_{t} \sim \mathrm{N}(0,1), \\
h_{t}=\mu+\phi\left(h_{t-1}-\mu\right)+\eta_{t}, & \eta_{t} \sim \mathrm{N}\left(0, \sigma_{\eta}^{2}\right)
\end{array}
$$

with $\theta=\left(\mu, \phi, \sigma_{\eta}^{2}\right)$ and $h=\left(h_{1}, \cdots, h_{T}\right)$. Applying Bayes' theorem we have

$$
p(\theta, h \mid y) \propto p(y \mid \theta, h) p(h \mid \theta) p(\theta) .
$$

Bayesian inference for the model parameters $\theta$ and the volatility states $h$ is based on the posterior distribution $p(\theta, h \mid y)$ which is proportional to the product of the likelihood function $p(y \mid \theta, h)$ specified by (31a), the conditional distribution of the volatility states $p(h \mid \theta)$ given by $31 \mathrm{~b}$, and the prior distribution $p(\theta)$. 
- Initialize $h^{(0)}, \mu^{(0)}, \phi^{(0)}$ and ${\sigma_{\eta}^{2}}^{(0)}$.

- For $g=1, \ldots, G$ :

1. For $t=1, \ldots, T$ :

Sample $h_{t}^{(g)}$ from $p\left(h_{t} \mid y, h_{<t}^{(g)}, h_{>t}^{(g-1)}, \mu^{(g-1)}, \phi^{(g-1)}, \sigma_{\eta}^{2(g-1)}\right)$.

2. Sample $\sigma_{\eta}^{2(g)}$ from $p\left(\sigma_{\eta}^{2} \mid y, h^{(g)}, \mu^{(g-1)}, \phi^{(g-1)}\right)$.

3. Sample $\phi^{(g)}$ from $p\left(\phi \mid y, h^{(g)}, \sigma_{\eta}^{2(g)}, \mu^{(g-1)}\right)$.

4. Sample $\mu^{(g)}$ from $p\left(\mu \mid y, h^{(g)}, \phi^{(g)}, \sigma_{\eta}^{2(g)}\right)$.

Figure 2: Single-move Gibbs sampler for the standard SV model

The model is completed by specifying the prior distributions for $\theta$. We assume that the model parameters are a priori independently distributed as follows:

$$
\begin{aligned}
p(\mu) & =\mathrm{N}\left(\alpha_{\mu}, \beta_{\mu}^{2}\right), \\
p(\phi) & =\mathrm{N}\left(\alpha_{\phi}, \beta_{\phi}^{2}\right) \mathbf{1}(-1,+1)(\phi), \\
p\left(\sigma_{\eta}^{2}\right) & =\mathbb{I} \mathbb{G}\left(\alpha_{\sigma}, \beta_{\sigma}\right),
\end{aligned}
$$

where $\mathbb{I} G(\cdot, \cdot)$ denotes an inverse-gamma distribution and $\mathrm{N}(a, b) \mathbf{1}(-1,+1)(x)$ defines a normal distribution with mean $a$, variance $b$, which is truncated between -1 and 1 . This rules out near unit-root behavior of $\phi$. The parameters $\alpha_{(\cdot)}$ and $\beta_{(\cdot)}$, characterizing the prior distributions, are called hyper-parameters, which are specified by the researcher.

Given the prior distributions, the conditional posteriors for the model parameters are derived as

$$
\begin{aligned}
& p\left(\mu \mid y, h, \phi, \sigma_{\eta}^{2}\right) \propto p\left(y \mid h, \mu, \phi, \sigma_{\eta}^{2}\right) p\left(h \mid \mu, \phi, \sigma_{\eta}^{2}\right) p(\mu), \\
& p\left(\phi \mid y, h, \sigma_{\eta}^{2}, \mu\right) \propto p\left(y \mid h, \mu, \phi, \sigma_{\eta}^{2}\right) p\left(h \mid \mu, \phi, \sigma_{\eta}^{2}\right) p(\phi) \\
& p\left(\sigma_{\eta}^{2} \mid y, h, \mu, \phi\right) \propto p\left(y \mid h, \mu, \phi, \sigma_{\eta}^{2}\right) p\left(h \mid \mu, \phi, \sigma_{\eta}^{2}\right) p\left(\sigma_{\eta}^{2}\right) .
\end{aligned}
$$

Since the volatility states $h$ subsume all information about $\left(\mu, \phi, \sigma_{\eta}^{2}\right)$, the full information likelihood function $p\left(y \mid h, \mu, \phi, \sigma_{\eta}^{2}\right)$ is a constant with respect to the model parameters, and thus can be omitted.

By successively conditioning we get

$$
p\left(h \mid \mu, \phi, \sigma_{\eta}^{2}\right)=p\left(h_{1} \mid \mu, \phi, \sigma_{\eta}^{2}\right) \prod_{t=1}^{T-1} p\left(h_{t+1} \mid h_{t}, \mu, \phi, \sigma_{\eta}^{2}\right),
$$

where $p\left(h_{t+1} \mid h_{t}, \mu, \phi, \sigma_{\eta}^{2}\right)$ is specified according to $31 \mathrm{~b}$. Moreover, inserting $p\left(\sigma_{\eta}^{2}\right), p(\phi)$, $p(\mu)$, given by $(33)$, and $p\left(h \mid \mu, \phi, \sigma_{\eta}^{2}\right)$, given by (35), into 34$)$, the full conditional posteriors can be reformulated, after eliminating constant terms, as (for details, see Appendix)

$$
\begin{aligned}
& p\left(\sigma_{\eta}^{2} \mid y, h, \mu, \phi\right) \propto \mathbb{I} \mathbb{G}\left(\hat{\alpha}_{\sigma}, \hat{\beta}_{\sigma}\right), \\
& p\left(\phi \mid y, h, \sigma_{\eta}^{2}, \mu\right) \propto \mathrm{N}\left(\hat{\alpha}_{\phi}, \hat{\beta}_{\phi}^{2}\right) \mathbf{1}(-1,+1)(\phi), \\
& p\left(\mu \mid y, h, \phi, \sigma_{\eta}^{2}\right) \propto \mathrm{N}\left(\hat{\alpha}_{\mu}, \hat{\beta}_{\mu}^{2}\right),
\end{aligned}
$$


where the hyper-parameters are estimated by

$$
\begin{aligned}
& \hat{\alpha}_{\sigma}=\alpha_{\sigma}+\frac{T}{2}, \\
& \hat{\beta}_{\sigma}=\beta_{\sigma}+\frac{1}{2}\left\{\sum_{t=1}^{T-1}\left(h_{t+1}-\mu-\phi\left(h_{t}-\mu\right)\right)^{2}+\left(h_{1}-\mu\right)^{2}\left(1-\phi^{2}\right)\right\}, \\
& \hat{\alpha}_{\phi}=\hat{\beta}_{\phi}^{2}\left\{\frac{\sum_{t=1}^{T-1}\left(h_{t+1}-\mu\right)\left(h_{t}-\mu\right)}{\sigma_{\eta}^{2}}+\frac{\alpha_{\phi}}{\beta_{\phi}^{2}}\right\}, \\
& \hat{\beta}_{\phi}^{2}=\left\{\frac{\sum_{t=1}^{T-1}\left(h_{t}-\mu\right)^{2}-\left(h_{1}-\mu\right)^{2}}{\sigma_{\eta}^{2}}+\frac{1}{\beta_{\phi}^{2}}\right\}^{-1}, \\
& \hat{\alpha}_{\mu}=\hat{\beta}_{\mu}^{2}\left\{\frac{h_{1}\left(1-\phi^{2}\right)+(1-\phi) \sum_{t=1}^{T-1}\left(h_{t+1}-\phi h_{t}\right)}{\sigma_{\eta}^{2}}+\frac{\alpha_{\mu}}{\beta_{\mu}^{2}}\right\}, \\
& \hat{\beta}_{\mu}^{2}=\left\{\frac{1-\phi^{2}+(T-1)(1-\phi)^{2}}{\sigma_{\eta}^{2}}+\frac{1}{\beta_{\mu}^{2}}\right\}^{-1} .
\end{aligned}
$$

Since it is possible to directly sample from the conditional posteriors, we obtain a straightforward (single-move) Gibbs sampler which breaks the joint posterior $p(\theta, h, y)$ into $T+3$ univariate conditional posteriors. The resulting Gibbs algorithm is summarized in figure 2, where the subscripts of $h_{<t}^{(\cdot)}$ and $h_{>t}^{(\cdot)}$ denote the periods before and after $t$ respectively.

The most difficult part of the estimation of SV models is to effectively sample the latent states $h_{t}$ from their full conditional posterior. In this context, an M-H A-R algorithm can be applied. Below we briefly illustrate a sampling procedure which is also used by Kim et al. (1998). In this context, Bayes' theorem implies

$$
\begin{aligned}
p\left(h_{t} \mid y, h_{-t}, \theta\right) & \propto p\left(y_{t} \mid h_{t}, \theta\right) p\left(h_{t} \mid h_{-t}, \theta\right), \\
& =\frac{1}{\sqrt{2 \pi \exp \left(h_{t}\right)}} \exp \left\{-\frac{y_{t}^{2}}{2 \exp \left(h_{t}\right)}\right\} p\left(h_{t} \mid h_{-t}, \theta\right), \\
& =f^{*}\left(y_{t}, h_{t}, \theta\right) p\left(h_{t} \mid h_{-t}, \theta\right),
\end{aligned}
$$

where, $h_{-t}$ denotes all elements of $h=\left(h_{1}, \cdots, h_{T}\right)$ excluding $h_{t}$. Exploiting the Markovian structure of the SV model we can derive

$$
p\left(h_{t} \mid h_{-t}, \theta\right)=p\left(h_{t} \mid h_{t-1}, h_{t+1}, \theta\right)=p_{N}\left(h_{t} \mid \alpha_{t}, \beta^{2}\right),
$$

where, $p_{N}(x \mid a, b)$ denotes the normal density function with mean $a$ and variance $b$, and

$$
\alpha_{t}=\mu+\frac{\phi\left\{\left(h_{t-1}-\mu\right)+\left(h_{t+1}-\mu\right)\right\}}{\left(1+\phi^{2}\right)}, \quad \beta^{2}=\frac{\sigma_{\eta}^{2}}{1+\phi^{2}} .
$$

An acceptance-rejection step is implemented exploiting the fact that $\exp \left(-h_{t}\right)$ is bounded by a linear function in $h_{t}$. By applying a Taylor expansion for $\exp \left(-h_{t}\right)$ around $\alpha_{t}$ we obtain

$$
\begin{aligned}
\log f^{*}\left(y_{t}, h_{t}, \theta\right) & \leq-\frac{1}{2} \log (2 \pi)-\frac{1}{2} h_{t}-\frac{y_{t}^{2}}{2}\left[\exp \left(-\alpha_{t}\right)\left\{1+\alpha_{t}-h_{t} \exp \left(-\alpha_{t}\right)\right\}\right] \\
& \stackrel{\text { def }}{=} \log g^{*}\left(y_{t}, h_{t}, \theta\right) .
\end{aligned}
$$


- For $t=1, \cdots, T$ :

1. Draw $h_{t}^{*}$ from $p_{N}\left(h_{t} \mid \alpha_{t}^{*}, \beta^{2}\right)$.

2. Draw $U$ from $\mathbb{U}[0,1]$.

3. If $U \leq f^{*}\left(y_{t}, h_{t}^{*}, \theta\right) / g^{*}\left(y_{t}, h_{t}^{*}, \theta\right)$

set $h_{t}=h_{t}^{*}$.

Else

go to step 1.

Figure 3: A-R method to sample the volatility states $h_{t}$

Since $p\left(h_{t} \mid h_{-t}, \theta\right)=p_{N}\left(h_{t} \mid \alpha_{t}, \beta^{2}\right)$, we have

$$
p\left(h_{t} \mid h_{-t}, \theta\right) f^{*}\left(y_{t}, h_{t}, \theta\right) \leq p_{N}\left(h_{t} \mid \alpha_{t}, \beta^{2}\right) g^{*}\left(y_{t}, h_{t}, \theta\right) .
$$

Then, the right-hand side of (52), after eliminating constant terms, can be represented by

$$
p_{N}\left(h_{t} \mid \alpha_{t}, \beta^{2}\right) g^{*}\left(y_{t}, h_{t}, \theta\right)=k \cdot p_{N}\left(h_{t} \mid \alpha_{t}^{*}, \beta^{2}\right),
$$

where $k$ is a real valued constant, and $p_{N}\left(h_{t} \mid \alpha_{t}^{*}, \beta^{2}\right)$ denotes a normal density with mean $\alpha_{t}^{*}=\alpha_{t}+\frac{\beta^{2}}{2}\left(y_{t}^{2} \exp \left\{-\alpha_{t}\right\}-1\right)$ and variance $\beta^{2}$.

Hence, since the target distribution, $p\left(h_{t} \mid h_{-t}, \theta\right) f^{*}\left(y_{t}, h_{t}, \theta\right)$, is bounded by $p_{N}\left(h_{t} \mid \alpha_{t}^{*}, \beta^{2}\right)$ up to a constant $k$, the acceptance-rejection method can be applied to sample $h_{t}$ from $p\left(h_{t} \mid y, h_{-t}, \theta\right)$ with acceptance probability

$$
P\left\{U \leq \frac{f^{* *}\left(y_{t}, h_{t}, \theta\right) p\left(h_{t} \mid h_{-t}, \theta\right)}{k p_{N}\left(h_{t} \mid \alpha_{t}^{*}, \beta^{2}\right)}\right\}=\frac{f^{* *}\left(y_{t}, h_{t}, \theta\right)}{g^{* *}\left(y_{t}, h_{t}, \theta\right)}
$$

where $U \sim \mathbb{U}[0,1]$. Figure 3 summarizes the A-R algorithm to sample the latent volatility states $h_{t}$.

\section{Empirical Illustrations}

\subsection{The data}

Below we will illustrate estimations of the standard SV model, the SVt model and the SVJ model based on time series of the DAX index, the Dow Jones index and the GBP/USD FX rate. All time series cover the period from 1 January, 1991 to 21 March, 2007. We use daily continuously compounded returns yielding 4,231 observations. Table 1 reports the mean, standard deviation, median, 10\%- and 90\%-quantiles, and the empirical skewness as well as kurtosis of the three series. All series reveal negative skewness and overkurtosis which is a common finding for financial returns.

\subsection{Estimation of SV Models}

The standard SV model is estimated by running the Gibbs and A-R M-H algorithm based on 25,000 MCMC iterations, where 5, 000 iterations are used as burn-in period. Table2 displays 


\begin{tabular}{cccccccc}
\hline \hline & Mean & SD & Median & $0.1-\mathrm{q}$ & $0.9-\mathrm{q}$ & Skewness & Kurtosis \\
\hline DAX & $3.7 \mathrm{e}-04$ & 0.013 & $5.0 \mathrm{e}-4$ & -0.021 & 0.006 & -0.295 & 7.455 \\
\hline Dow Jones & $3.6 \mathrm{e}-04$ & 0.009 & $3.0 \mathrm{e}-4$ & -0.009 & 0.008 & -0.230 & 8.276 \\
\hline GBP/USD & $3.6 \mathrm{e}-06$ & 0.005 & $<1.0 \mathrm{e}-9$ & -0.006 & 0.009 & -0.126 & 5.559 \\
\hline \hline
\end{tabular}

Table 1: Summary statistics for daily returns of the DAX index, the Dow Jones index, and the GBP/USD exchange rate from 01/01/1991 to 21/03/2007.

the choice of the prior distributions and the hyper-parameters as well as the resulting prior mean and standard deviation.

Table 3 shows the sample mean (MEAN), the sample standard deviation (SD), the timeseries standard errors (ts-SE), and the 95\%-credibility interval (CI) based on $G=20,000$ MCMC replications. The time-series standard errors give an estimate of the variation that is expected in computing the mean of the MC replications and is computed as $S D / \sqrt{n}$. As a rule of thumb, Geweke (1992) suggests to choose $G$ such that the time series standard error is less than approximately $5 \%$ of the sample standard deviation.

\begin{tabular}{ccccc}
\hline \hline Prior Distribution & \multicolumn{2}{c}{ Hyper-Parameters } & Mean & S.D. \\
\hline$p(\mu)=\mathrm{N}\left(\alpha_{\mu}, \beta_{\mu}^{2}\right)$ & $\alpha_{\mu}=0$ & $\beta_{\mu}=100$ & 0 & 10 \\
$p(\phi)=\mathrm{N}\left(\alpha_{\phi}, \beta_{\phi}^{2}\right) I_{(-1,+1)}(\phi)$ & $\alpha_{\mu}=0$ & $\beta_{\mu}=100$ & 0 & 1 \\
$p\left(\sigma_{\eta}^{2}\right)=\mathcal{I} \mathcal{G}\left(\alpha_{\sigma}, \beta_{\sigma}\right)$ & $\alpha_{\sigma}=2.5$ & $\beta_{\sigma}=0.025$ & 0.167 & 0.024 \\
\hline \hline
\end{tabular}

Table 2: Prior distributions, hyper-parameters, and implied prior means as well as standard deviations for the standard $S V$ model.

Since the three time series reveal similar properties, we concentrate on the results for DAX index returns. The volatility process is highly persistent as indicated by an estimate of $\phi$ of 0.989 . This near-to-unit-root behavior is a quite typical finding for financial return series and is consistent with the commonly observed volatility clustering. The estimated (smoothed) volatility states are computed by

$$
\hat{h}_{t}=\frac{1}{G-g_{1}} \sum_{g=g_{1}+1}^{G} \exp \left(h_{t}^{(g)} / 2\right),
$$

where $h_{t}^{(g)}$ denotes the realizations of the Markov chain stemming from the M-H A-R algorithm illustrated in the previous section, and $g_{1}$ is the burn-in period. The resulting plots of the smoothed volatilities are shown in figure 4. It is nicely illustrated that the estimated latent volatility closely mimics the movements of $\left|y_{t}\right|$ supporting the idea of using absolute or squared returns as (noisy) proxies for $h_{t}$. 


\begin{tabular}{|c|c|c|c|c|}
\hline Parameter & Mean & $\mathrm{SD}$ & ts-SE & $95 \% \mathrm{CI}$ \\
\hline \multicolumn{5}{|l|}{ DAX } \\
\hline$\mu$ & -8.942 & 0.192 & $1.5 \mathrm{e}-3$ & $(-9.327,-8.565)$ \\
\hline$\phi$ & 0.989 & 0.002 & $2.0 \mathrm{e}-4$ & $(0.983,0.994)$ \\
\hline$\sigma_{\eta}$ & 0.115 & 0.009 & $1.0 \mathrm{e}-3$ & $(0.096,0.137)$ \\
\hline \multicolumn{5}{|l|}{ Dow Jones } \\
\hline$\mu$ & -9.471 & 0.171 & $1.3 \mathrm{e}-3$ & $(-9.810,-9.142)$ \\
\hline$\phi$ & 0.990 & 0.003 & $2.0 \mathrm{e}-4$ & $(0.984,0.995)$ \\
\hline$\sigma_{\eta}$ & 0.087 & 0.010 & $1.1 \mathrm{e}-3$ & $(0.069,0.108)$ \\
\hline \multicolumn{5}{|l|}{ GBP/USD } \\
\hline$\mu$ & -10.238 & 0.649 & $4.3 \mathrm{e}-3$ & $(-10.519,-9.997)$ \\
\hline$\phi$ & 0.993 & 0.002 & $2.0 \mathrm{e}-4$ & $(0.988,0.997)$ \\
\hline$\sigma_{\eta}$ & 0.041 & 0.006 & $8.0 \mathrm{e}-4$ & $(0.029,0.054)$ \\
\hline
\end{tabular}

Table 3: Estimation results for the standard SV model.
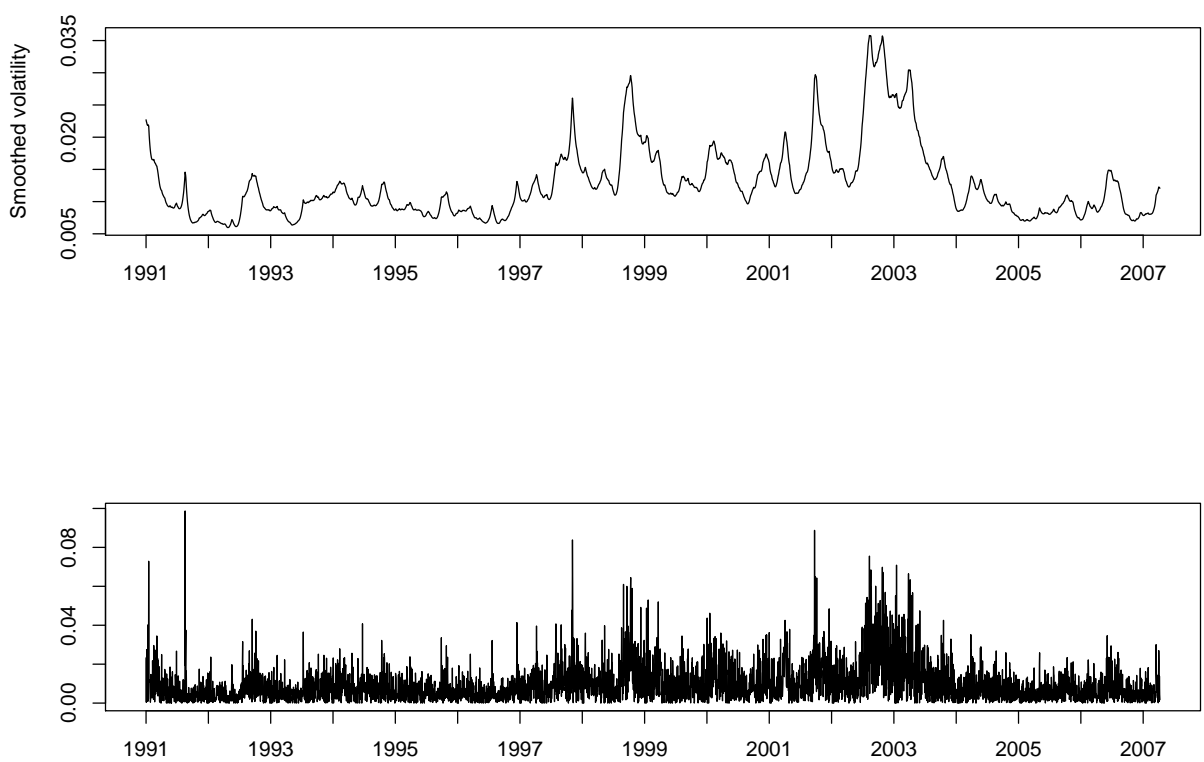

Figure 4: Top: Smoothed estimates of $h_{t}$. Bottom: Absolute returns, $\left|y_{t}\right|$.

Misspecification tests are implemented based on the standardized innovations, $y_{t} \exp \left(-\hat{h}_{t} / 2\right)$ which should be i.i.d. Applying Ljung-Box tests and ARCH tests (Engle 1982) shown in figure 5 yield $p$-values of 0.094 and 0.023 , respectively. For the BDS independence test we find a $p$-value of 0.011 . The corresponding plot of the standardized innovations as well as ACF plots of standardized innovations and squared standardized innovations are given by graphs (a), (c) and (d), respectively, in figure 5. The standardized innovations reveal a big outlier on 19/08/1991 where the DAX index dropped from 1653.33 to 1497.93. 
Such a behavior is not easily captured by a continuous distribution for $h_{t}$ and requires accounting for jumps. Nevertheless, though it is evident that the model is obviously not flexible enough to completely explain the volatility dynamics, the diagnostics indicate a quite satisfying dynamic performance. This is particularly true when the parameter parsimony of the model is taken into account.

It is not surprising that the model is unable to capture the distributional properties of the returns. We observe that the standard SV model with a model implied kurtosis of 5.74 is not able to fully explain the over-kurtosis in the data. This is confirmed by the Jarque-Bera normality test and the QQ plot revealing departures from normality mainly stemming from extreme innovations.

(a) Standardized innovations

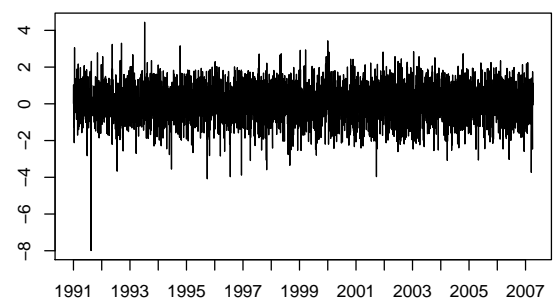

(c) ACF of standardized innovations

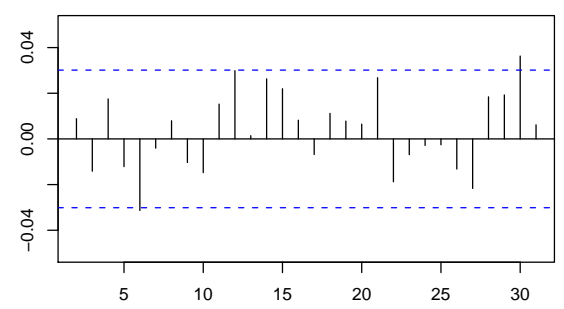

(c) QQ plot of standardized innovations

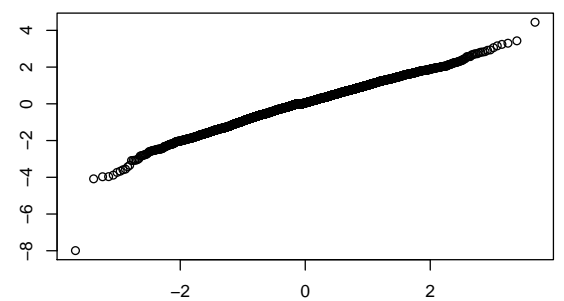

(d) ACF of squared standardized innovations

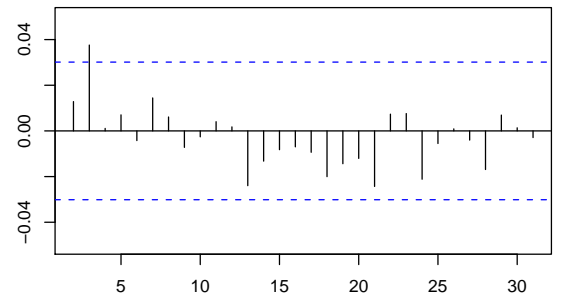

Figure 5: Time series plot, QQ plot and autocorrelogram of (squared) standardized innovations.

Finally, the results of convergence diagnostics are reported in table 4. All parameters pass both the Geweke's z-scores test and the Heidelberg-Welch's stationarity and half-width tests indicating a proper convergence of the Markov chain to its invariant distribution.

Table 5 shows the estimation results based on the SVt and SVJ model. For the sake of brevity and given that we have qualitatively similar findings for the other return series, we focus only on DAX index returns. We obtain an estimate of the degrees of freedom in the SVt model of about $\hat{\nu}=12.44$ indicating the presence of fat-tailedness in the data and a clear misspecification of the standard (Gaussian) SV model. The estimates for the SVJ model reveal a daily average jump size of about $\hat{\alpha}_{k}=0.005 \%$ with estimated standard 


\begin{tabular}{|c|c|c|c|c|c|c|}
\hline \multirow[t]{2}{*}{ Parameter } & \multicolumn{2}{|c|}{ Z-score Test } & \multicolumn{4}{|c|}{ Stationarity and Half-Width Test } \\
\hline & z-score & p-value & p-value & Mean & Half-width & Ratio \\
\hline$\mu^{S V}$ & 0.199 & 0.843 & 0.645 & -8.895 & 0.003 & -0.001 \\
\hline$\phi^{S V}$ & 0.032 & 0.972 & 0.897 & 0.928 & 0.001 & 0.001 \\
\hline$\sigma_{u}^{S V}$ & -0.413 & 0.686 & 0.979 & 0.329 & 0.003 & 0.009 \\
\hline
\end{tabular}

Table 4: Convergence Diagnostics

Note: The half-width test is passed if the corresponding ratio is less than 0.01 .

\begin{tabular}{ccccc}
\hline \hline Parameter & Mean & SD & ts-SE & $95 \%$ CI \\
\hline The SVt model: & & & & \\
$\mu$ & -9.201 & 0.230 & $2.3 \mathrm{e}-3$ & $(-9.663,-8.752)$ \\
$\phi$ & 0.991 & 0.002 & $1.0 \mathrm{e}-4$ & $(0.985,0.995)$ \\
$\sigma_{\eta}$ & 0.117 & 0.012 & $1.1 \mathrm{e}-3$ & $(0.095,0.145)$ \\
$\nu$ & 12.443 & 1.812 & $2.3 \mathrm{e}-1$ & $(9.600,16.923)$ \\
\hline$\mu$ & & & & \\
$\phi$ & -9.107 & $2.3 \mathrm{e}-01$ & $1.8 \mathrm{e}-03$ & $(-9.568,-8.663)$ \\
$\sigma_{\eta}$ & 0.991 & $2.7 \mathrm{e}-03$ & $2.1 \mathrm{e}-04$ & $(0.984,0.995)$ \\
$\alpha_{k}$ & 0.124 & $1.3 \mathrm{e}-02$ & $1.4 \mathrm{e}-03$ & $(0.101,0.153)$ \\
$\sqrt{\beta_{k}}$ & -0.005 & $2.9 \mathrm{e}-05$ & $1.8 \mathrm{e}-07$ & $(-0.005,-0.004)$ \\
$\kappa$ & 0.029 & $6.5 \mathrm{e}-03$ & $8.7 \mathrm{e}-04$ & $(0.020,0.045)$ \\
\hline The SVJ model: & 0.010 & $3.9 \mathrm{e}-03$ & $3.1 \mathrm{e}-04$ & $(0.003,0.019)$ \\
\hline \hline
\end{tabular}

Table 5: Estimation results for the SVt and SVJ model based on DAX index returns.

deviation $\sqrt{\hat{\beta}_{k}}=0.029$. Estimates of $\kappa$ reveal an average probability of observing a jump of about $1 \%$ on a daily basis. This implies that on average a jump in returns may occur on average every 100 trading days.

Figure 6 depicts the QQ plots of the normalized innovations based on the standard SV model (left), the SVt model (middle), and the SVJ model (right). It is shown that the inclusion of Student-t errors improves the distributional properties of the model only slightly. Actually, we observe that both the basic SV and the SVt model are not able to capture extreme observations in the tails of the distribution. In contrast, the SVJ model turns out to be more appropriate to accommodate outliers. This result indicates the importance of allowing returns to be driven by a jump component.

\section{Conclusions}

In this paper we reviewed the basic SV model as well as its various extensions. The economic implications behind such models are prominent for both researchers and practitioners. Furthermore, we also illustrate the major principles of MCMC based statistical inference for 

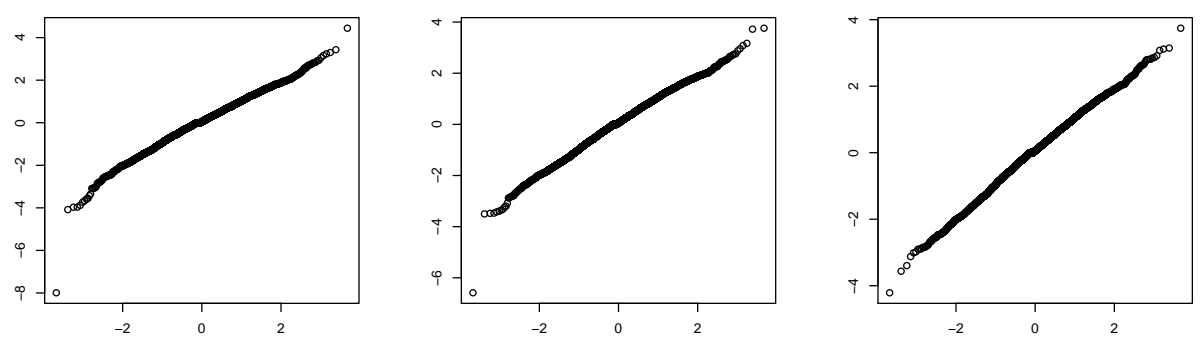

Figure 6: QQ plots of normalized innovations based on the standard SV model (left), the SVt model (middle), and the SVJ model (right).

SV-type models. Bayesian inference is based on the combination of subjective and objective information. Hence, it provides a way to incoprorate our personal belief into statistically models. Finally, our empirical study indicates that both the basic SV and the SVt model are not able to capture extreme observations in the tails of the distribution, and it is important to allow returns to be driven by a jump component.

\section{Appendix}

\section{Derivation of the Conditional Posterior Distributions}

Using Bayes' theorem, the conditional posterior distribution of $\sigma_{\eta}^{2}$ is given by

$$
p\left(\sigma_{\eta}^{2} \mid y, h, \mu, \phi\right) \propto p\left(y \mid h, \mu, \phi, \sigma_{\eta}^{2}\right) p\left(h \mid \mu, \phi, \sigma_{\eta}^{2}\right) p\left(\sigma_{\eta}^{2}\right)
$$

By assuming $\sigma_{\eta}^{2}$ to follow an inverse-gamma distribution and successively conditioning on $p\left(h \mid \mu, \phi, \sigma_{\eta}^{2}\right)$, we obtain

$$
p\left(\sigma_{\eta}^{2} \mid y, h, \mu, \phi\right) \propto p\left(h_{1} \mid \mu, \phi, \sigma_{\eta}^{2}\right) \prod_{t=1}^{T-1} p\left(h_{t+1} \mid h_{t}, \mu, \phi, \sigma_{\eta}^{2}\right) \mathbb{I} \mathbb{G}\left(\sigma_{\eta}^{2} \mid \alpha_{\sigma}, \beta_{\sigma}\right),
$$

where the density function $p\left(h_{t+1} \mid h_{t}, \mu, \phi, \sigma_{\eta}^{2}\right)$ is given by $(1 \mathrm{~b})$. 
After eliminating all constant terms with respect to $\sigma_{\eta}^{2}$, we obtain

$$
\begin{aligned}
& p\left(\sigma_{\eta}^{2} \mid y, h, \mu, \phi\right) \\
& \propto \exp \left[-\frac{\left(h_{1}-\mu\right)^{2}\left(1-\phi^{2}\right)}{2 \sigma_{\eta}^{2}}-\frac{\sum_{t=1}^{T-1}\left\{h_{t+1}-\mu-\phi\left(h_{t}-\mu\right)\right\}^{2}}{2 \sigma_{\eta}^{2}}\right] \\
& \quad \times\left(\frac{1}{\sigma_{\eta}^{2}}\right)^{\frac{T}{2}} \frac{\left(\beta_{\sigma}\right)^{\alpha_{\sigma}} e^{-\beta_{\sigma} / \sigma_{\eta}^{2}}}{\Gamma\left(\alpha_{\sigma}\right)\left(\sigma_{\eta}^{2}\right)^{\alpha_{\sigma}+1}} \\
& \propto \exp \left[-\frac{\beta_{\sigma}+\frac{1}{2}\left(h_{0}-\mu\right)^{2}\left(1-\phi^{2}\right)+\frac{1}{2} \sum_{t=1}^{T-1}\left\{h_{t+1}-\mu-\phi\left(h_{t}-\mu\right)\right\}^{2}}{\sigma_{\eta}^{2}}\right] \\
& \quad \times\left(\frac{1}{\sigma_{\eta}^{2}}\right)^{\left(\alpha_{\sigma}+\frac{T}{2}\right)+1} .
\end{aligned}
$$

It is easy to see that the posterior density $p\left(\sigma_{\eta}^{2} \mid y, h, \mu, \phi\right)$ is proportional to an inverse-gamma density. Consequently, we have

$$
p\left(\sigma_{\eta}^{2} \mid y, h, \mu, \phi\right) \propto \mathbb{I} \mathbb{G}\left(\hat{\alpha}_{\sigma}, \hat{\beta}_{\sigma}\right)
$$

where,

$$
\begin{aligned}
& \hat{\alpha}_{\sigma}=\alpha_{\sigma}+\frac{T}{2} \\
& \hat{\beta}_{\sigma}=\beta_{\sigma}+\frac{1}{2}\left(h_{1}-\mu\right)^{2}\left(1-\phi^{2}\right)+\frac{1}{2} \sum_{t=1}^{T-1}\left\{h_{t+1}-\mu-\phi\left(h_{t}-\mu\right)\right\}^{2} .
\end{aligned}
$$

Mimicking the proceeding for $\sigma_{\eta}^{2}$ we can derive the conditional posteriors for $\mu$ and $\phi$ in a similar way. Then, we obtain

$$
\begin{aligned}
p\left(\mu \mid y, h, \phi, \sigma_{\eta}^{2}\right) & \propto p\left(h \mid \mu, \phi, \sigma_{\eta}^{2}\right) p(\mu), \\
& \propto p\left(h_{1} \mid \mu, \phi, \sigma_{\eta}^{2}\right) \prod_{t=1}^{T-1} p\left(h_{t+1} \mid h_{t}, \mu, \phi, \sigma_{\eta}^{2}\right) \mathrm{N}\left(\alpha_{\mu}, \beta_{\mu}\right), \\
& \propto \exp \left(-\frac{1}{2}\left[\mu^{2}\{\underbrace{\frac{1-\phi^{2}+(T-1)(1-\phi)^{2}}{\sigma_{\eta}^{2}}+\frac{1}{\beta_{\mu}^{2}}}_{A}\}\right.\right. \\
& \left.\left.-2 \mu\{\underbrace{\frac{h_{1}\left(1-\phi^{2}\right)+(1-\phi) \sum_{t=1}^{T-1}\left(h_{t+1}-\phi h_{t}\right)}{\sigma_{\eta}^{2}}+\frac{\alpha_{\mu}}{\beta_{\mu}^{2}}}_{B}\}\right]\right), \\
& \propto \mathrm{N}\left(\frac{B}{A}, \frac{1}{A}\right)
\end{aligned}
$$


and

$$
\begin{aligned}
p\left(\phi \mid y, h, \sigma_{\eta}^{2}, \mu\right) & \propto p\left(h \mid \mu, \phi, \sigma_{\eta}^{2}\right) p(\phi), \\
& \propto p\left(h_{1} \mid \mu, \phi, \sigma_{\eta}^{2}\right) \prod_{t=1}^{T-1} p\left(h_{t+1} \mid h_{t}, \mu, \phi, \sigma_{\eta}^{2}\right) \mathrm{N}\left(\alpha_{\phi}, \beta_{\phi}^{2}\right) \infty_{(-1,+1)}(\phi), \\
& \propto \exp \left(-\frac{1}{2}\left[\phi^{2}\{\underbrace{\frac{-\left(h_{1}-\mu\right)^{2}+\sum_{t=1}^{T-1}\left(h_{t}-\mu\right)^{2}}{\sigma_{\eta}^{2}}+\frac{1}{\beta_{\phi}^{2}}}_{C}\}\right.\right. \\
& \left.\left.\quad-2 \phi\{\underbrace{\frac{\sum_{t=1}^{T-1}\left(h_{t+1}-\mu\right)\left(h_{t}-\mu\right)}{\sigma_{\eta}^{2}}+\frac{\alpha_{\phi}}{\beta_{\phi}^{2}}}_{D}\}\right]\right) \infty_{(-1,+1)}(\phi), \\
& \propto \mathrm{N}\left(\frac{D}{C}, \frac{1}{C}\right) \infty_{(-1,+1)}(\phi) .
\end{aligned}
$$

\section{References}

Andersen, T. G., Bollerslev, T., Diebold, F.X., \& Labys, P. (2003). Modeling and Forecasting Realized Volatility, Econometrica 71: 579-625.

Arteche, J. (2004). Gaussian semiparametric estimation in long memory in stochastic volatility and signal plus noise models, Journal of Econometrics 119: 131-154.

Baillie, R. T. (1996). Long memory processes and fractional integration in econometrics, Journal of Econometrics 73: 5-59.

Bates, D. (2000). Post-'87 Crash fears in S\&P 500 futures options, Journal of Econometrics 9: $69-107$.

Beran, J. (1994). Statistics for Long-Memory Processes, Chapman and Hall, Boca Raton.

Besag, J. (1974). Spatial interaction and the statistical analysis of lattice systems, Journal of the Royal Statistical Society Series B 36: 192-236.

Black, F. (1976). Studies of stock price volatility changes, Proceedings of the $19^{7} 6$ Meetings of the American Statistical Association, Business and Economical Statistics Section $177-181$.

Bollerslev, T. \& Mikkelsen, H. O. (1996). Modeling and pricing long memory in stock market volatility, Journal of Econometrics 73: 151-184.

Breidt, F. J., Carto, N., \& de Lima, P. (1998). The detection and estimation of long memory in stochastic volatility models, Journal of Econometrics 83: 325-348. 
Brockwell, A. E. (2005). Likelihood-based analysis of a class generalized long-memory time series models, Journal of Time Series Analysis 28: 386-407.

Bauwens, L., Lubrano, M., \& Richard, J. F. (1999). Bayesan Inference in Dynamic Econometric Models, Oxford University Press \& Sons, New York.

Campbell, J. Y. \& Hentschel, L. (1992). No news is good news: An asymmetric model of changing volatility in stock returns, Journal of Financial Economics 31: 281-318.

Chib, S., Nardari, F., \& Shephard, N. (2002). Markov Chain Monte Carlo methods for stochastic volatility models, Journal of Econometrics 108: 281-316.

Cowles, M. K. \& Carlin, B. P. (1996). Markov Chain Monte Carlo convergence diagnostics: A comparative review, Journal of the American Statistical Association 91: 883-905.

Clark, P.K. (1973). A subordinated stochastic process model with finite variance for speculative prices,", Econometrica 41: 135-156.

Danielsson, J. (1994). Stochastic volatility in asset prices: Estimation with simulated maximum likelihood, Journal of Econometrics 64: 375-400.

Duffie, D., Pan, J., \& Singleton, K. (2000). Transform analysis and asset pricing for affine jump-diffusions, Econometrica 68: 1343-1376.

Engle, R. F. (1982). Autoregressive conditional heteroscedasticity with estimates of the variance of United Kingdom inflation, Econometrica 50: 987-1007.

Eraker, B., Johannes, M., \& Polson, N. (2003). The impact of jumps in volatility and returns, Journal of Finance 58: 1269-1300.

French, K. R., Schwert, G. W., \& Stambaugh, R. F. (1987). Expected stock returns and volatility, Journal of Financial Economics 19: 3-29.

Frieze, A., Kannan, R., \& Polson N. (1994). Sampling from log-concave distributions, Annals of Applied Probability 4: 812-834.

Gallant, A.R., Hsie, D., \& Tauchen G. (1997). Estimation of stochastic volatility models with diagnostics, Journal of Econometrics 81: 159-192.

Geman, S. \& Geman D. (1984). Stochastic relaxation, Gibbs distributions, and the Bayesian restoration of images, IEEE Transactions on Pattern Analysis and Machine Intelligence 6: 721-741. 
Geweke, J. (1992). Evaluating the accuracy of sampling-based approaches to the calculation of posterior moments, in J. M. Bernardo, J. O. Berger, A. P. Dawid, A. F. M. Smith (ed.), Bayesian Statistics 4, Oxford University Press, Amsterdam.

Ghysels, E., Harvey, A. C., \& Renault E. (1996). Stochastic volatility, in G. S. Maddala and C. R. Rao (ed.), Handbook of Statistics 14, Statistical Methods in Finance, NorthHolland, Amsterdam.

Granger, C. W. \& Joyeux, R. (1980). An introduction to long-memory time series models and fractional differencing, Journal of Time Series Analysis 1: 15-29.

Greenberg, E. (2008). Introduction to Bayesian Econometrics, Cambridge University Press, New York.

Hammersley, J. \& Clifford, P. (1971). Markov fields on finite graphs and lattices, Unpublished Manuscipt.

Harvey, A. C. (1998). Long-memory in stochastic volatility, in J. Knight and S. E. Satchell (ed.), Forecasting Volatility in Financial Markets, Butterworth-Heinemann, London, pp. 203-226.

Harvey, A. C., Ruiz, E., \& Shephard, N. (1994). Multivariate stochastic variance models, Review of Economic Studies 61: 247-264.

Harvey, A. C. \& Shephard, N. (1996). The estimation of an asymmetric stochastic volatility model for asset returns, Journal of Business and Economics Statistics 14: 429-434.

Hastings, W. K. (1970). Monte Carlo sampling methods using Markov chains and their applications, Biometrika 57: 97-109.

Jacquier, E., Polson, N. G., \& Rossi, P. E. (1994). Bayesian analysis of stochastic volatility models (with discussion), Journal of Business and Economic Statistics 12: 371-417.

Jacquier, E., Polson, N. G., \& Rossi, P. E. (2004). Bayesian analysis of stochastic volatility models with fat-tails and correlated errors, Journal of Econometrics 122: 185-212.

Kim, S., Shephard, N., \& Chib, S. (1998). Stochastic volatility: Likelihood inference and comparison with ARCH models, Review of Economic Studies 65: 361-393.

Koop, G. (2006). Bayesian Econometrics, Wiley-Interscience, London.

Liesenfeld, R. \& Richard, J. F. (2003). Univariate and multivariate stochastic volatility models: Estimation and diagnostics, Journal of Empirical Finance 10: 505-531. 
Liesenfeld, R. \& Jung, R. C. (2000). Stochastic volatility models: Conditional normality versus heavy-tailed distributions, Journal of Applied Econometrics 15: 137-160.

Metropolis, N., Rosenbluth, A., Rosenbluth, M., Teller, A., \& Teller, E. (1953). Equations of state calculations by fast computing machines, Journal of Chemical Physics 21: 10871092.

Melino, A. \& Turnbull, S. M. (1990). Pricing foreign currency options with stochastic volatility, Journal of Econometrics 45: 239-265.

Sandmann, G. \& Koopman, S. J. (1998). Estimation of stochastic volatility models via Monte Carlo maximum likelihood, Journal of Econometrics 87: 271-301.

Taylor, S. J. (1982). Financial returns modelled by the product of two stochastic processes, a study of daily sugar prices 1961-79, in O. D. Anderson (ed.), Time Series Analysis: Theory and Practice 1, North-Holland, Amsterdam, pp. 203-226.

Tierney, L. (1994). Markov Chains for exploring posterior distributions, The Annals of Statistics 22: 1701-1762. 


\section{SFB 649 Discussion Paper Series 2008}

For a complete list of Discussion Papers published by the SFB 649, please visit http://sfb649.wiwi.hu-berlin.de.

001 "Testing Monotonicity of Pricing Kernels" by Yuri Golubev, Wolfgang Härdle and Roman Timonfeev, January 2008.

002 "Adaptive pointwise estimation in time-inhomogeneous time-series models" by Pavel Cizek, Wolfgang Härdle and Vladimir Spokoiny, January 2008.

003 "The Bayesian Additive Classification Tree Applied to Credit Risk Modelling" by Junni L. Zhang and Wolfgang Härdle, January 2008.

004 "Independent Component Analysis Via Copula Techniques" by Ray-Bing Chen, Meihui Guo, Wolfgang Härdle and Shih-Feng Huang, January 2008.

005 "The Default Risk of Firms Examined with Smooth Support Vector Machines" by Wolfgang Härdle, Yuh-Jye Lee, Dorothea Schäfer and Yi-Ren Yeh, January 2008.

006 "Value-at-Risk and Expected Shortfall when there is long range dependence" by Wolfgang Härdle and Julius Mungo, Januray 2008.

007 "A Consistent Nonparametric Test for Causality in Quantile" by Kiho Jeong and Wolfgang Härdle, January 2008.

008 "Do Legal Standards Affect Ethical Concerns of Consumers?" by Dirk Engelmann and Dorothea Kübler, January 2008.

009 "Recursive Portfolio Selection with Decision Trees" by Anton Andriyashin, Wolfgang Härdle and Roman Timofeev, January 2008.

010 "Do Public Banks have a Competitive Advantage?" by Astrid Matthey, January 2008.

011 "Don't aim too high: the potential costs of high aspirations" by Astrid Matthey and Nadja Dwenger, January 2008.

012 "Visualizing exploratory factor analysis models" by Sigbert Klinke and Cornelia Wagner, January 2008.

013 "House Prices and Replacement Cost: A Micro-Level Analysis" by Rainer Schulz and Axel Werwatz, January 2008.

014 "Support Vector Regression Based GARCH Model with Application to Forecasting Volatility of Financial Returns" by Shiyi Chen, Kiho Jeong and Wolfgang Härdle, January 2008.

015 "Structural Constant Conditional Correlation" by Enzo Weber, January 2008.

016 "Estimating Investment Equations in Imperfect Capital Markets" by Silke Hüttel, Oliver Mußhoff, Martin Odening and Nataliya Zinych, January 2008.

017 "Adaptive Forecasting of the EURIBOR Swap Term Structure" by Oliver Blaskowitz and Helmut Herwatz, January 2008.

018 "Solving, Estimating and Selecting Nonlinear Dynamic Models without the Curse of Dimensionality" by Viktor Winschel and Markus Krätzig, February 2008.

019 "The Accuracy of Long-term Real Estate Valuations" by Rainer Schulz, Markus Staiber, Martin Wersing and Axel Werwatz, February 2008.

020 "The Impact of International Outsourcing on Labour Market Dynamics in Germany" by Ronald Bachmann and Sebastian Braun, February 2008.

021 "Preferences for Collective versus Individualised Wage Setting" by Tito Boeri and Michael C. Burda, February 2008.

\section{SFB 649, Spandauer Straße 1, D-10178 Berlin http:/ / sfb649.wiwi.hu-berlin.de}

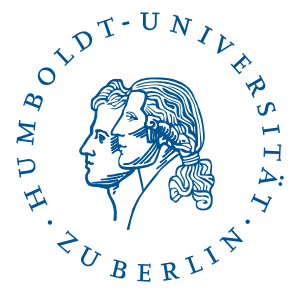


022 "Lumpy Labor Adjustment as a Propagation Mechanism of Business Cycles" by Fang Yao, February 2008.

023 "Family Management, Family Ownership and Downsizing: Evidence from S\&P 500 Firms" by Jörn Hendrich Block, February 2008.

024 "Skill Specific Unemployment with Imperfect Substitution of Skills" by Runli Xie, March 2008.

025 "Price Adjustment to News with Uncertain Precision" by Nikolaus Hautsch, Dieter Hess and Christoph Müller, March 2008.

026 "Information and Beliefs in a Repeated Normal-form Game" by Dietmar Fehr, Dorothea Kübler and David Danz, March 2008.

027 "The Stochastic Fluctuation of the Quantile Regression Curve" by Wolfgang Härdle and Song Song, March 2008.

028 "Are stewardship and valuation usefulness compatible or alternative objectives of financial accounting?" by Joachim Gassen, March 2008.

029 "Genetic Codes of Mergers, Post Merger Technology Evolution and Why Mergers Fail" by Alexander Cuntz, April 2008.

030 "Using R, LaTeX and Wiki for an Arabic e-learning platform" by Taleb Ahmad, Wolfgang Härdle, Sigbert Klinke and Shafeeqah Al Awadhi, April 2008.

031 "Beyond the business cycle - factors driving aggregate mortality rates" by Katja Hanewald, April 2008.

032 "Against All Odds? National Sentiment and Wagering on European Football" by Sebastian Braun and Michael Kvasnicka, April 2008.

033 "Are CEOs in Family Firms Paid Like Bureaucrats? Evidence from Bayesian and Frequentist Analyses" by Jörn Hendrich Block, April 2008.

034 "JBendge: An Object-Oriented System for Solving, Estimating and Selecting Nonlinear Dynamic Models" by Viktor Winschel and Markus Krätzig, April 2008.

035 "Stock Picking via Nonsymmetrically Pruned Binary Decision Trees" by Anton Andriyashin, May 2008.

036 "Expected Inflation, Expected Stock Returns, and Money Illusion: What can we learn from Survey Expectations?" by Maik Schmeling and Andreas Schrimpf, May 2008.

037 "The Impact of Individual Investment Behavior for Retirement Welfare: Evidence from the United States and Germany" by Thomas Post, Helmut Gründl, Joan T. Schmit and Anja Zimmer, May 2008.

038 "Dynamic Semiparametric Factor Models in Risk Neutral Density Estimation" by Enzo Giacomini, Wolfgang Härdle and Volker Krätschmer, May 2008.

039 "Can Education Save Europe From High Unemployment?" by Nicole Walter and Runli Xie, June 2008.

040 "Solow Residuals without Capital Stocks" by Michael C. Burda and Battista Severgnini, August 2008.

041 "Unionization, Stochastic Dominance, and Compression of the Wage Distribution: Evidence from Germany" by Michael C. Burda, Bernd Fitzenberger, Alexander Lembcke and Thorsten Vogel, March 2008

042 "Gruppenvergleiche bei hypothetischen Konstrukten - Die Prüfung der Übereinstimmung von Messmodellen mit der Strukturgleichungsmethodik" by Dirk Temme and Lutz Hildebrandt, June 2008.

043 "Modeling Dependencies in Finance using Copulae" by Wolfgang Härdle, Ostap Okhrin and Yarema Okhrin, June 2008.

044 "Numerics of Implied Binomial Trees" by Wolfgang Härdle and Alena Mysickova, June 2008.

\section{SFB 649, Spandauer Straße 1, D-10178 Berlin http:/ / sfb649.wiwi.hu-berlin.de}

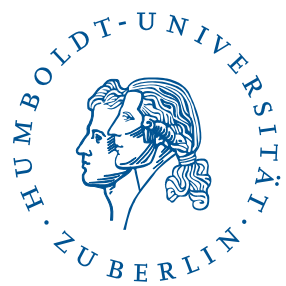


045 "Measuring and Modeling Risk Using High-Frequency Data" by Wolfgang Härdle, Nikolaus Hautsch and Uta Pigorsch, June 2008.

046 "Links between sustainability-related innovation and sustainability management" by Marcus Wagner, June 2008.

047 "Modelling High-Frequency Volatility and Liquidity Using Multiplicative Error Models" by Nikolaus Hautsch and Vahidin Jeleskovic, July 2008.

048 "Macro Wine in Financial Skins: The Oil-FX Interdependence" by Enzo Weber, July 2008.

049 "Simultaneous Stochastic Volatility Transmission Across American Equity Markets" by Enzo Weber, July 2008.

050 "A semiparametric factor model for electricity forward curve dynamics" by Szymon Borak and Rafał Weron, July 2008.

051 "Recurrent Support Vector Regreson for a Nonlinear ARMA Model with Applications to Forecasting Financial Returns" by Shiyi Chen, Kiho Jeong and Wolfgang K. Härdle, July 2008.

052 "Bayesian Demographic Modeling and Forecasting: An Application to U.S. Mortality" by Wolfgang Reichmuth and Samad Sarferaz, July 2008.

053 "Yield Curve Factors, Term Structure Volatility, and Bond Risk Premia" by Nikolaus Hautsch and Yangguoyi Ou, July 2008.

054 "The Natural Rate Hypothesis and Real Determinacy" by Alexander MeyerGohde, July 2008.

055 "Technology sourcing by large incumbents through acquisition of small firms" by Marcus Wagner, July 2008.

056 "Lumpy Labor Adjustment as a Propagation Mechanism of Business Cycle" by Fang Yao, August 2008.

057 "Measuring changes in preferences and perception due to the entry of a new brand with choice data" by Lutz Hildebrandt and Lea Kalweit, August 2008.

058 "Statistics E-learning Platforms: Evaluation Case Studies" by Taleb Ahmad and Wolfgang Härdle, August 2008.

059 "The Influence of the Business Cycle on Mortality" by Wolfgang $\mathrm{H}$. Reichmuth and Samad Sarferaz, September 2008.

060 "Matching Theory and Data: Bayesian Vector Autoregression and Dynamic Stochastic General Equilibrium Models" by Alexander Kriwoluzky, September 2008.

061 "Eine Analyse der Dimensionen des Fortune-Reputationsindex" by Lutz Hildebrandt, Henning Kreis and Joachim Schwalbach, September 2008.

062 "Nonlinear Modeling of Target Leverage with Latent Determinant Variables - New Evidence on the Trade-off Theory" by Ralf Sabiwalsky, September 2008.

063 "Discrete-Time Stochastic Volatility Models and MCMC-Based Statistical Inference" by Nikolaus Hautsch and Yangguoyi Ou, September 2008.

\section{SFB 649, Spandauer Straße 1, D-10178 Berlin} http:/ / sfb649.wiwi.hu-berlin.de 